\title{
Determination of Elastic Parameters due a Dynamic Hertzian Contact
}

\author{
Juan Pablo Villacreses ${ }^{1,2}$, Bernardo Caicedo ${ }^{1}$, Silvia Caro ${ }^{1}$, Fabricio Yepez ${ }^{2}$, Joel Sebastián Puebla ${ }^{2}$ \\ ${ }^{1}$ Department of Civil and Environmental Engineering, Universidad de los Andes \\ Bogota, Colombia \\ jp.villacreses@uniandes.edu.co; bcaicedo@uniandes.edu.co \\ ${ }^{2}$ Department of Civil Engineering, Universidad San Francisco de Quito \\ Quito, Ecuador \\ fyepez@usfq.edu.ec; jspueblar@estud.usfq.edu.ec
}

\section{Extended Abstract}

An earthquake is a natural spontaneous phenomenon, representing a threat not only to people but also to the economy and the infrastructure of any country [1]. Effects of seismic activity could be shrunk by improving the uncertainty associated with the measurement of soil parameters [2]. Dynamic behavior of soils is related to some properties such as shear modulus $(G)$, Young's modulus (E) and damping ratio $(\lambda)[3]$. There are several techniques to measure these parameters, but some of them are too expensive for daily projects. Consequently, professional practitioners need a wider variety of tests to measure elastic properties and use them as input in computational engineering models.

This research is based on the Hertzian Contact Theory. Specifically, shear and Young modulus of the soil were computed using a wave produced by an impact of a steel sphere. The impact caused by a short transient force generates deformation on the soil surface. As a result, deformation produces two types of waves: body waves and surface waves. Body wave's velocity (i.e., P-wave and S-wave) is related to the material elastic properties [4]. Consequently, P and S-wave's travel time through a medium was measured using two sensors located at the top and at the bottom surfaces of the soil samples.

An accelerometer and a high-frequency transducer were used for travel time measurement in this test. In detail, a highfrequency ICP ${ }^{\circledR}$ accelerometer was placed on the top sample surface located at $5 \mathrm{~mm}$ from the impact point. This accelerometer uses a frequency range between $0.0016 \mathrm{kHz}$ and $30 \mathrm{kHz}$. At the same time, either a $\mathrm{P}$ or $\mathrm{S}$-wave transducer was placed in the middle of the sample's cross section bottom surface. Specifically, a Pundit Lab Transducer with a frequency range from $20 \mathrm{kHz}$ to $500 \mathrm{kHz}$ was used for P-waves measurements. Whereas, a shear wave transducer, model V151 -RB was used for S-Waves.

Three clay samples were prepared with different volumetric properties. The clay had a plasticity index of $17.9 \%$, a liquid limit of $35 \%$ and a specific gravity of 2.59 . The process of soil preparation was made in two phases: water addition, and sieving process. In detail, a No. 8 sieve was used and the passed material was stored in a hermetic plastic bag until the mixture was homogenous. Samples with different densities were prepared by compacting and using three different water content according to the Standard Proctor Method, ASTM D 698 [5]. The samples had a density of $1.98 \mathrm{~g} / \mathrm{cm} 3,2.00$ $\mathrm{g} / \mathrm{cm} 3$ and $1.96 \mathrm{~g} / \mathrm{cm} 3$ and a water content of $19 \%, 21 \%$ and $22 \%$, respectively. Compacted samples were extracted from the mold and tested without any confinement. Each sample had been tested five times to get a reliable result.

A steel sphere of $6.4 \mathrm{~mm}$ of diameter and $1.04 \mathrm{~g}$ of weight was dropped from a controlled height of $5 \mathrm{~cm}$. Using this procedure and the measured values of waves velocities from the Proctor samples, Young and shear modulus were calculated. Another cylindrical samples of $13.00 \mathrm{~mm}$ of diameter and $45.00 \mathrm{~mm}$ of height were constructed with the same volumetric properties. These samples were tested using a Reomether TA Instruments Ar 2000, in which the equipment applied a cyclic strain of $1.00 \times 10^{-4}$. The Rheometer TA has been used to measure the shear modulus of the soil. The procedure is conducted according to the research conducted by Villacreses et al. 2020 [6]. These values are used to compare the measured values obtained through the Hertzian contact wave propagation. The results of the two sets of samples were compared, and the maximum measured difference was around $25 \%$. This small difference between the results suggest that this experimental technique could be used as an alternative method to measure dynamic soil properties at small deformations. 


\section{References}

[1] C. H. Scholz, "Earthquakes and Faulting: Self-Organized Critical Phenomena with a Characteristic Dimension," in Riste T., Sherrington D. (eds) Spontaneous Formation of Space-Time Structures and Criticality. NATO ASI Series (Series C: Mathematical and Physical Sciences), vol 349, Springer, Dordrecht, 1991.

[2] Zi-Jun Cao, Yu Wang, Dian-Qing Li, "Site-Specific Characterization of Soil Properties Using Multiple Measurements from Different Test Procedures at Different Locations - A Bayesian Sequential Updating Approach," Engineering Geology, 2016.

[3] S. Shankar, A. Murali and A. Dey, "Parameters Influencing Dynamic Soil Properties: A Review Treatise," International Journal of Innovative Research in Science, Engineering and Technology. vol. 3, pp. 47 - 49, 2014.

[4] T. Pradhan, "Finite Element Modeling of Impact-Generated Stress Wave Propagation in Concrete Plates for NonDestructive Evaluation," Theses and Dissertations, no. 1597, 2015.

[5] ASTM D698 - 12. (2012). Standard Test Methods for Laboratory Compaction Characteristics of Soil Using Standard Effort $(12,400 \mathrm{ft}-\mathrm{lbf} / \mathrm{ft} 3(600 \mathrm{kN}-\mathrm{m} / \mathrm{m} 3))$.

[6] J. P. Villacreses, B. Caicedo, S. Caro, F. Yépez, “A novel procedure to determine shear dynamic modulus and damping ratio for partial saturated compacted fine-grained soils, Soil Dynamics and Earthquake Engineering, pp. 2 - 3, 2020. 